\section{Military Technical College Kobry El-Kobbah, Cairo, Egypt}

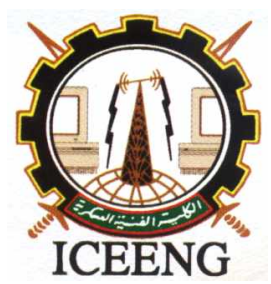

\author{
$6^{\text {th }}$ International Conference \\ on Electrical Engineering \\ ICEENG 2008
}

\title{
Adaptive threshold mapping technique for moving target detector (MTD) in modern radar
}

\author{
By \\ Alaa El-Din Sayed Hafez* $\quad$ Ahmed Ibrahim Salem*
}

\section{Abstract:}

In this paper we propose a new adaptive threshold mapping (ATM) technique for moving target detector (MTD) in modern radar. This technique is proposed to reduce the false alarms results from weather clutter and interference with relatively high Doppler frequency shift and MTD is don't able to suppress it. Such technique is based on controlling the receiver sensitivity according the status of the output signal at the post detection. Also the technique is control the signal processing path with relatively high probability of detection. The Simulation results show that this technique is a powerful solution to keep the post detection output with high probability of detection and minimum probability of false alarm rate compared with tradition MTD without (ATM) technique.

\section{Keywords:}

Radar, MTD, Clutter Map

* Faculty of Air Defense, Alexandria 


\section{Introduction:}

The addition of digital processing to radars today increases the radar's sensitivity. This additional sensitivity requires more robust clutter processing to maintain optimal system performance. Adaptive detection techniques rely on homogeneity in range and sets threshold using secondary data collected from neighboring cells [1-5]. Actual statistical characterization of clutter background is critical to the design of efficient target detection and identification algorithms for radar systems. Several clutter map algorithms [6-12] are proposed to improve the radar signal detection. The proposed adaptive threshold mapping technique to reduce the false alarms results from weather clutter and interference with relatively high Doppler frequency shift and MTD is don't able to suppress it. This technique is based on controlling the receiver sensitivity according to the status of the output signal at the post detection. The post detection output averaged, hence if exceeding primary threshold value the adaptive threshold map control the sensitivity of the receiver by attenuating the received signal. If the averaged values exceeding a secondary threshold value the circuit adopt a fixed threshold to the receiver to reduce the false alarm rate.

\section{System Description.:}

The simulated moving target detector system (MTD) is shown in figure (1). The MTD is an enhanced configuration of moving-target indicator (MTI) that combines a series of features to improve clutter rejection and target detection. The pre-detected signal passed through digital attenuator and fixed threshold circuit, which control the amplitude level and the threshold of the pre-detected signal. The processed signal is directed to phase detector, which split the detected signal into inphase and quadrature phase signal as a function of the Doppler frequency. The echo signal is processed through Doppler filter bank to get eight narrow frequency bands through the Doppler frequency as shown in figure (2). The first and last frequency band include the echoes from stationary ground clutter and sea clutter while the other frequency bands include the echoes of the true targets, interference, weather clutter, and angel clutter. The CFAR (Constant-False-Alarm-Rate) processing circuit is a very important part in SP. Its main function is to suppress moving clutter arises from weather, such as cloud, rain and so on, because it is receives input from the average value of the filter bank outputs from \# 1 to \#6, which contains the information of the moving targets only, ignoring the filter bank output number \#0 and \#7, which contains the information of the stationary clutter. . In an automatic detection system of radar, the false-alarm-rate is very sensitive to the ratio between the threshold and the clutter intensity. Generally, if the threshold is fixed, the false-alarm-rate will increase much more when the clutter level goes up with several 
decibels. Because of that, the detection system would be overloaded and could not make any correct decision. Thus, some measures should be taken to keep a constant falsealarm-rate (CFAR).

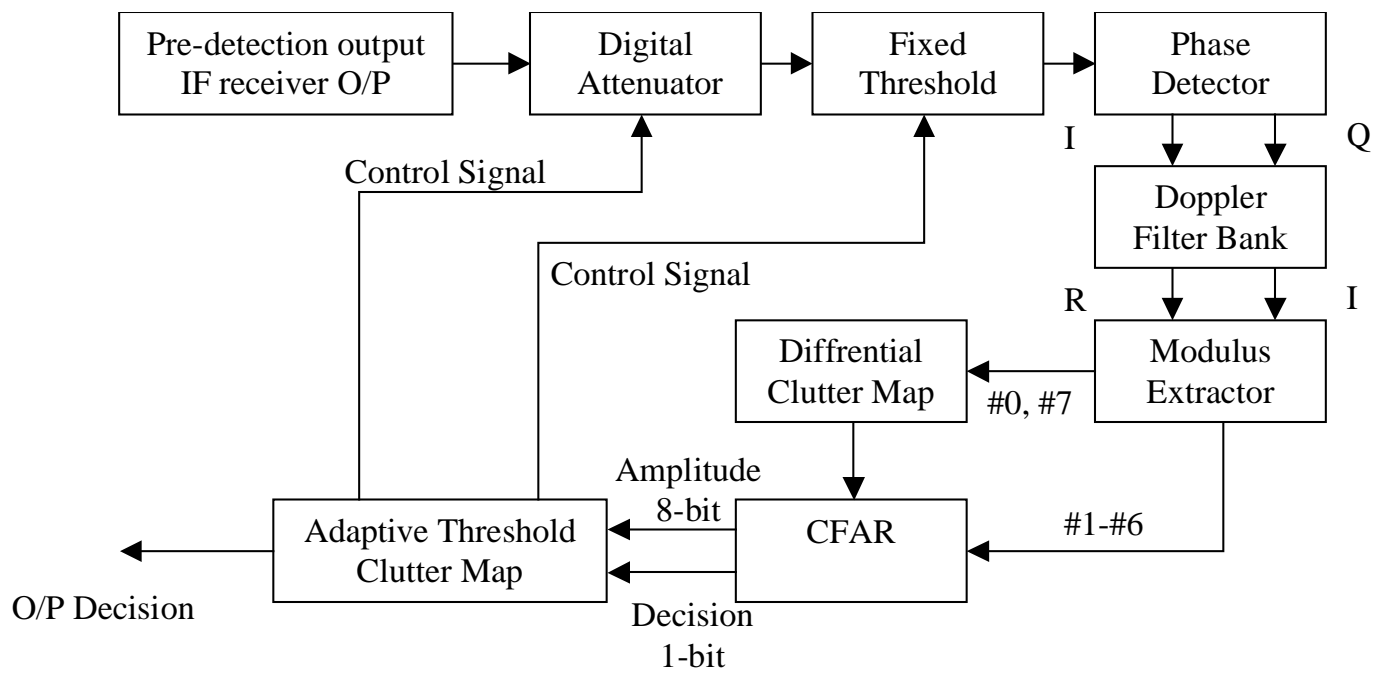

Figure (1): MTD block diagram

The Doppler filter is the heart of the MTD signal processor, it is sometimes called FIR (finite impulse response) filter and undertakes the work of frequency-spectrum analysis [13]. According to the theory of Doppler effect, there is always a frequency shift in the echo from a moving target. This shift is directly proportional to the radial speed of the target and is called Doppler frequency shift in practice. Its representative is [13],

$$
f_{d}=\frac{2 v_{r}}{\lambda}
$$

where, $v_{r}$ stands for the radial speed of the target and $\lambda$ the wavelength of the radar transmitting signal. Assuming the weight factor for the filter No. K (K \#filter),

$$
w_{k}(n)=\exp \left(-\frac{j 2 \pi k}{N} n\right), \mathrm{n}=0,1, \ldots \ldots, \mathrm{N}-1
$$

the output will be,

$$
Y(k)=\sum_{n=0}^{N-1} f(n) \cdot w_{k}(n), \mathrm{k}=0,1, \ldots \ldots, \mathrm{N}-1
$$



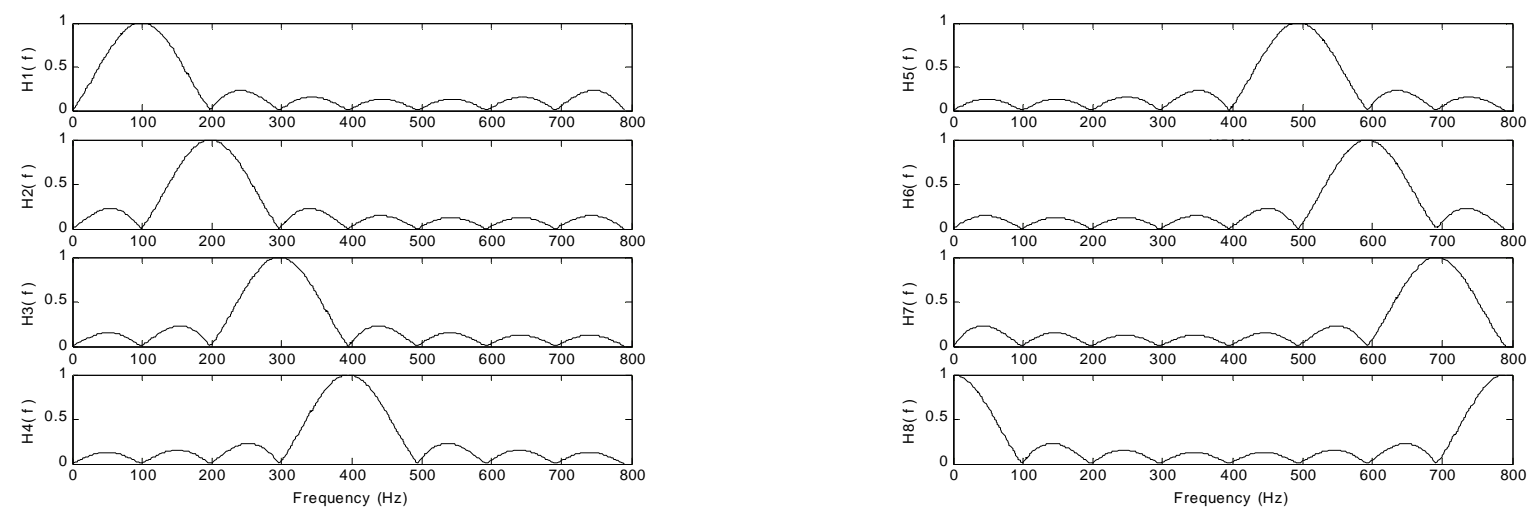

Figure (2): The characteristics of Doppler filter bank

The CFAR treatment is based on the understanding of the statistical characteristics of interference, i.e. the interference (clutter or noise) voltage after the envelope detection is a statistically homogeneous random variable with a Rayleigh distribution.

Its probability density function (PDF) is defined as,

$$
P(v)=\frac{v}{\sigma^{2}} \exp \left[-\frac{v^{2}}{2 \sigma^{2}}\right] \quad \mathrm{v} \geq 0
$$

where $\sigma$ represents the interference standard deviation before the envelope detection. If variable $\mathrm{v}$ is normalized by deviation $\sigma$, that is to say, set $\mathrm{u}=\mathrm{v} / \sigma$, then the PDF of new variable u may be written,

$$
P(v)=u \exp \left[-\frac{u^{2}}{2}\right] \quad \mathrm{v} \geq 0
$$

As seen clearly from the above formula, the function $\mathrm{P}(\mathrm{u})$ is not dependent on the interference deviation directly in this case. The detection of variable $u$ through a fixed threshold will not result in the change of false-alarm-rate with the interference intensity. Hence, the problem of CFAR processing has been simplified to that of variable normalization ( $\mathrm{v}$ is divided by $\sigma$ ), and from statistics it has been known that the mean value of $\mathrm{v}$ is equal to $\sqrt{\pi} \sigma / \sqrt{2}$, and smooth filtering is equivalent to averaging (getting the mean value). Therefore, CFAR treatment can be realized by such a way as Figure (3). Referring to the figure, an input sample is taken from each range cell. The samples of the cell under test (CUT) and its neighboring cells (reference cells) are obtained from a tapped delay line in an analog implementation. There are N/2 reference cells on the left, and equal ones on the right of the CUT too. The inputs from both the N/2 cells are averaged separately, resulting two averages. Then the larger one is chosen to be an estimate of the interference mean value [14],

$$
\bar{v}=\operatorname{Avg}\left(M_{1}, M_{2}\right)
$$




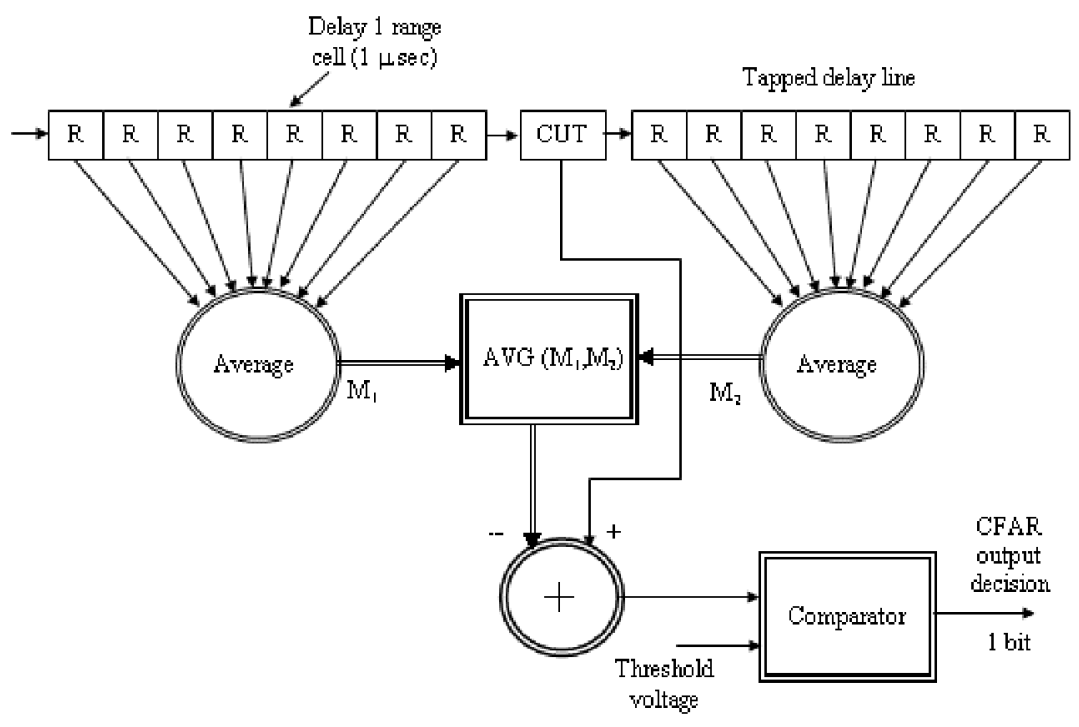

Figure (3): Functional diagram of cell averaging CFAR

$$
\bar{v}=A V G\left(\frac{2}{N} \sum_{i=0}^{\frac{N}{2}-1} V_{i}, \frac{1}{N} \sum_{\frac{N}{2}+1}^{N} V_{i}\right)
$$

Finally, when we subtract the estimate from the CUT input, the CFAR processing comes into effect. In addition, it is necessary to give reason for choosing the larger estimate from the right and the left of the CUT. This is mainly based on the following consideration: As soon as a target enters into a strong clutter region, there is strong clutter in the front $\mathrm{N} / 2$ cells, but no or a little clutter in the back ones. If we averaged the inputs from all the cells simply, the result would not be a good estimate of the interference mean value, and yet must be a little too small, which would lead the falsealarm-rate too large at once. After the method of "Cell Averaging" is adopted, the above case can be avoided. On the contrary, when the target leaves the strong clutter region once more, this kind of method may result too small false-alarm-rate. The differential clutter processes the output of first and last Doppler filter which register the present scan and the previous one for processing using a differential clutter map filter as shown in figure (4) [15]. Each clutter map cell estimate is updated by averaging some clutter density measurements, the averaging process may be the moving average,

$$
N_{k}(c)=\frac{1}{L} \sum_{j=k-L+1}^{k} \mu_{j}(c)
$$




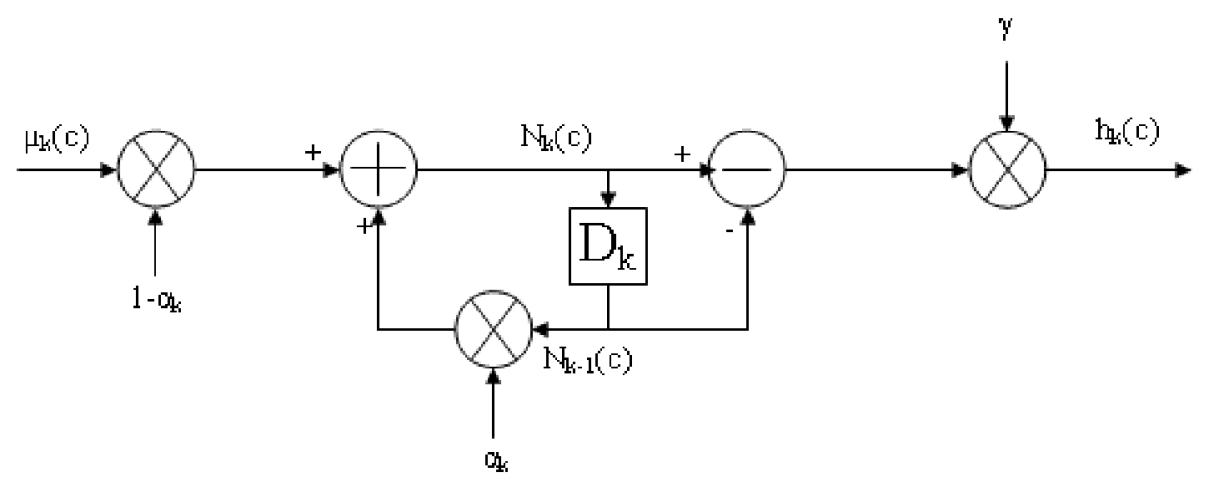

Figure (4): The schematic diagram of differential clutter map

where $\mathrm{c}$ denotes the clutter map cell, $\mathrm{k}$ denotes the time of measurement arrival, $\mathrm{k}$ is the scan index. The clutter density measurement to be averaged in cell at time $\mathrm{k}$ by $\mu_{\mathrm{k}}(\mathrm{c})$ and $\mathrm{N}_{\mathrm{k}}(\mathrm{c})$ denotes the averaged value. Averaging time constant is denoted by $\mathrm{L}$. The other possibility is true auto regressive filter for averaging.

$$
N_{k}(c)=\alpha_{k} N_{k-1}(c)+\left(1-\alpha_{k}\right) \mu_{k}(c)
$$

The differential filter to be applied is,

$$
h_{k}(c)=\gamma \frac{d N_{k}(c)}{d k}
$$

where $\gamma$ is a differential declaration factor of the filter. The numerical representation of the differential filter is,

$$
h_{k}(c)=\gamma\left(N_{k}(c)+N_{k-1}(c)\right)
$$

\section{Adaptive threshold map Algorithm:}

The algorithm used for this type of processing is based on measuring the running average of the signal and control the receiver sensitivity. This control process depending on the amplitude of the signal average value. If the average value exceeded the first threshold, ATM control the level of the received signal using digital attenuator until the average value exceeded a second threshold, ATM begins to adopt a fixed threshold level to the received signal. The algorithm used for the adaptive threshold map (ATM) is shown in figure (5). 


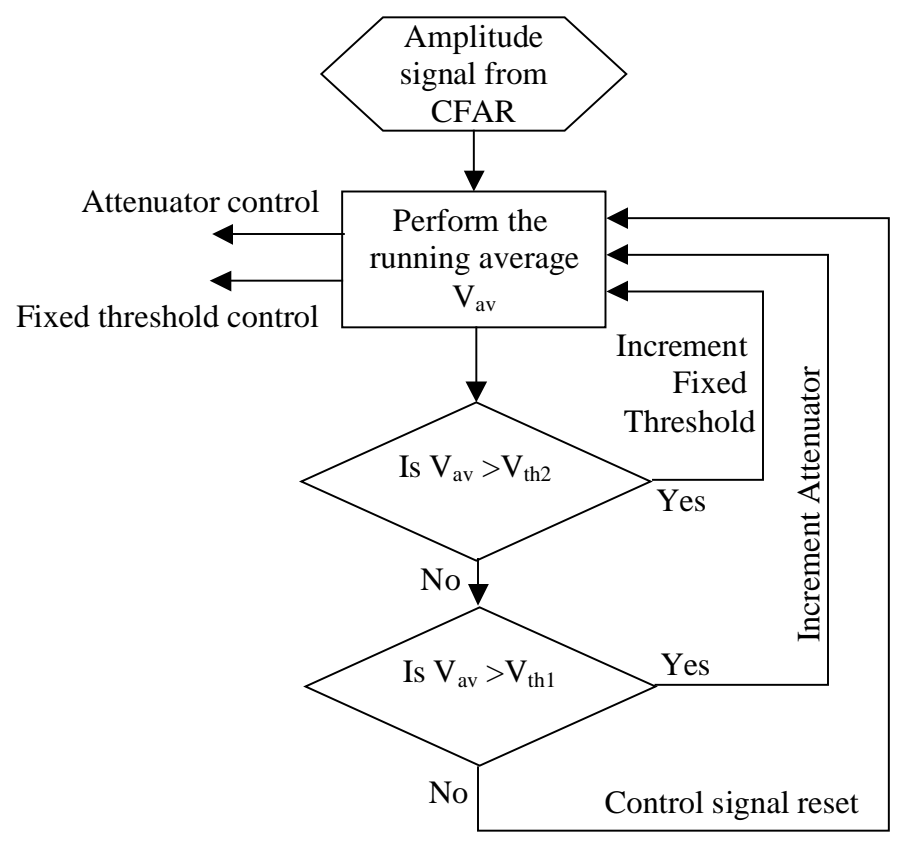

Figure (5): Adaptive threshold map algorithm

\section{Simulation Results:}

The proposed architecture is simulated under software matlab environment. The simulated signals contain noise with spectrum of normal distribution and include moving targets, stationary ground and sea clutter. The output of the system is tested under low and high threshold level of CA-CFAR processor. Figure (6) shows the probability of false alarm rate versus attenuation at different CFAR thresholds. it is clear that introduce the attenuation reduce the probability of false alarm rate. At low CFAR threshold $\mathrm{P}_{\mathrm{fa}}$ increased then the system need more attenuation to reduce $\mathrm{P}_{\mathrm{fa}}$. increase the CFAR threshold significantly reduce the signal attenuation. Figure (7) show that the fixed threshold introduced to the pre-detected signal improve significantly the probability of false alarm rate $\mathrm{P}_{\mathrm{fa}}$ and keep high probability of detection.

\section{Conclusion:}

The proposed architecture is an adaptive threshold map that monitors the status of the output signal from CFAR and control the receiver sensitivity for high probability of false alarm rate the map control a fixed threshold adopted to the pre-detected signal. For medium probability of false alarm the map control the attenuator to attenuate the predetected signal. The Simulation results show that this technique is a powerful solution to keep the post detection output with high probability of detection and minimum probability of false alarm rate compared with tradition MTD without (ATM) technique 


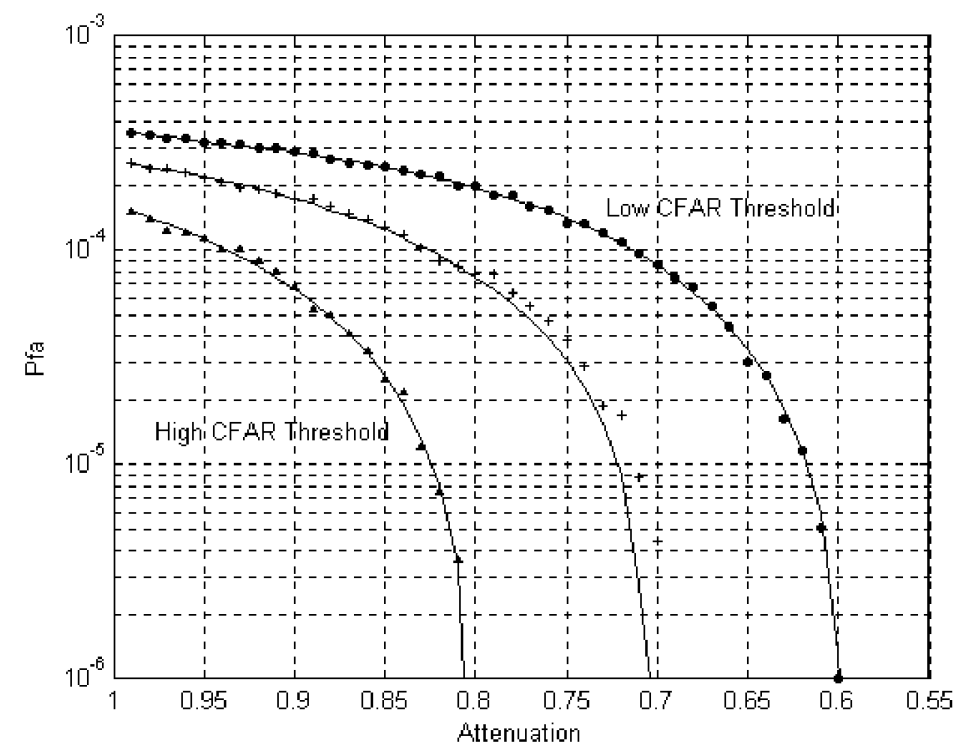

Figure (6): probability of false alarm versus Attenuation

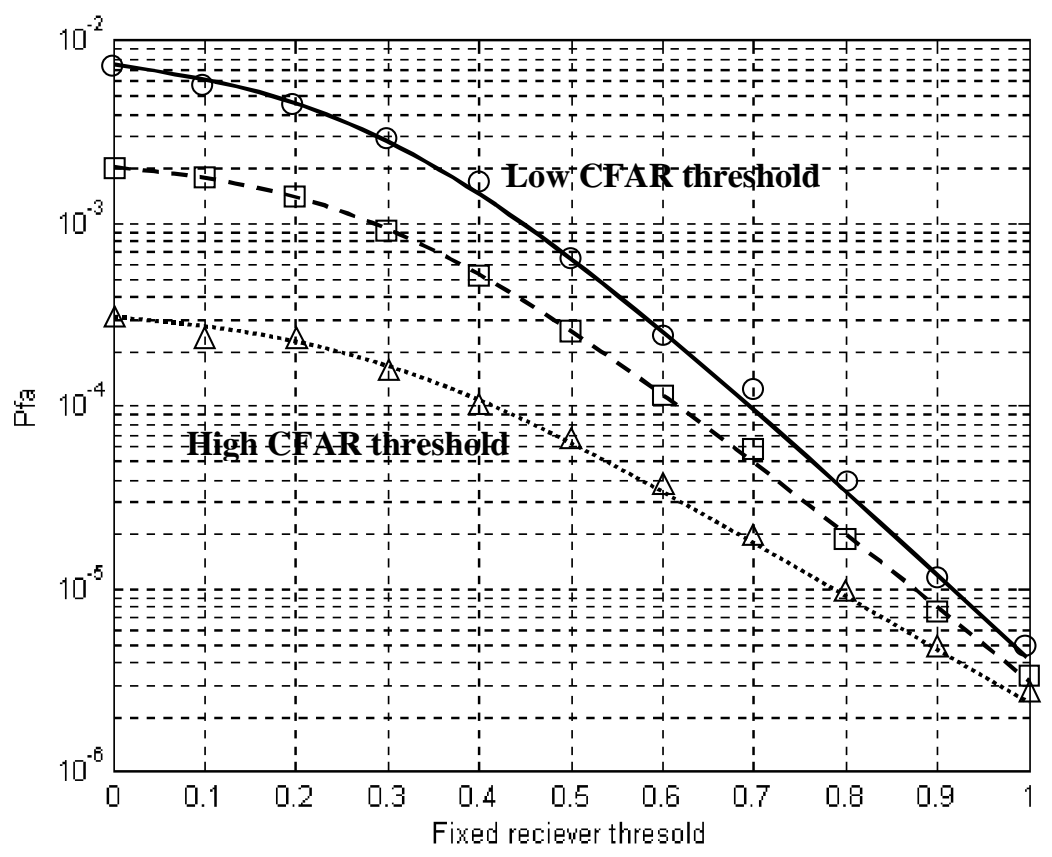

Figure (7) : probability of false alarm versus fixed threshold 


\section{References:}

[1] Blum, R. S., and Kassam, S. A."Distributed Cell-Averaging CFAR Detection in Dependent Sensors." IEEE Transactions on Aerospace and Electronic Systems, vol. 41, No. 2, pp. 513-522, March 1995.

[2] Salem, A. I., Elfaramawy, N., and El-Badawy, E. A.; "CFAR Different Schemes Behavior and Detection Performance of Moving Targets." $8^{\text {th }}$ International World multi-Conference on Systemics, Cybernetics, and Informatics, SCI-2004, Orlando, Florida, USA. pp. 295 - 300, July 18-21, 2004.

[3] Santos Lopez Estrada, Rene Cumplido parra, Cesar Torres Huitzil, "A Hybrid Approatch for target detection Using CFAR Algorithm and Image Processing", Proceedings of the Fifth Mexician International Conference in Computer Science (ENC'04), 2004.

[4] Darko Musicki, Sofa Suvorova, Mark Morelande and Bill Moran, "Clutter Map and Target Tracking" $7^{\text {th }}$ International Conference on Information Fusion, 2005.

[5] Zhi-guo. Qu, Xian-si Tan, Hong. Wang, Gang. He, "A CFAR Based on Statistics of Cell Under Test”, Radar 2006. CIE'06 International Conference on, China, Oct. 2006.

[6] Darko Musicki, Subhash Challa,and Sfia Suvorova, "Automatic Track Initiation of Manoevring Target in Clutter", IEEE Proceeding conferences, 2004.

[7] L. Ted Rogers, Lee J. Wagner, Peter Gerstoft, Jeffrey L.Korlik and Michael Jablecki, "Refractivity from Clutter (RFC)", IEEE Proceeding conferences, 2004.

[8] Ruth Rosenholtz, Yuanzhen Li, Jonathan Mansfield, and Zhenlan Jin, "Feature Congestion : AMeasure of Display Clutter", IEEE Proceeding conferences, 2005.

[9] Marc Berenguer, Daniel Sempere Torres, Rafael Sanchez-Diezma, and Ramon Pascual, "Identification of Clutter Echoes Using A Fuzzy Logic Technique", IEEE Proceeding conferences, 2004.

[10]Bencheikh, M. L. Magaz. B., Hamadouche. M., Belouchrani A., "Analysis and Real Time Implementation of a Clutter map CFAR detector with Noncoherent Integration", International Radar Symposium, 2006, IRS 2006, Poland, May, 2006.

[11] Song Wanjie, Liu Juntao, Tao Haihong, Wu Shunjun, "The principle and Performance analysis of profile clutter map", Radar 2006. CIE'06 International Conference on, China, Oct. 2006.

[12]Zhang Tao, Boult Terrance E. Johnson R. C., "Two Thresholds are better than one", Computer vision and pattern recognition, 2007, CVPR'07, IEEE conference on, Minneapolis, MN, USA, June, 2007.

[13] Merrill I. Skolnik, "Radar Handbook", McGraw-Hill, New York, 2007.

[14] Simon Kingsley, Shaun Quegan, "Understanding Radar Systems", McGraw-Hill, New York, 1992.

[15] Alaa S. Hafez, "A New Differential Clutter Map Processing in Modern Radar", The $24^{\text {th }}$ National Radio Science Conference NRSC'2007,Egypt, March 13-15, 2007. 